\section{OPEN ACCESS}

Edited by:

Jane Zhen Liang,

Shenzhen University, China

Reviewed by:

Linling $\mathrm{Li}$,

Shenzhen University, China

Binlong Zhang,

Guang'anmen Hospital, China Academy of Chinese Medical

Sciences, China

*Correspondence:

Zhenlan Jin

jinzl@uestc.edu.cn

Ling Li

liling@uestc.edu.cn

Specialty section:

This article was submitted to

Perception Science,

a section of the journal

Frontiers in Neuroscience

Received: 17 March 2021

Accepted: 11 May 2021

Published: 17 June 2021

Citation:

Li W, Xie K, Ngetich RK, Zhang J,

Jin Z and Li L (2021) Inferior Frontal

Gyrus-Based Resting-State

Functional Connectivity and Medium

Dispositional Use of Reappraisal

Strategy. Front. Neurosci. 15:681859.

doi: 10.3389/fnins.2021.681859

\title{
Inferior Frontal Gyrus-Based Resting-State Functional Connectivity and Medium Dispositional Use of Reappraisal Strategy
}

Wenjuan Li, Ke Xie, Ronald K. Ngetich, Junjun Zhang, Zhenlan Jin* and Ling Li*

MOE Key Laboratory for Neuroinformation, High-Field Magnetic Resonance Brain Imaging Key Laboratory of Sichuan Province, Center for Psychiatry and Psychology, School of Life Sciences and Technology, University of Electronic Science and Technology of China, Chengdu, China

The previous neuroimaging functional connectivity analyses have indicated that the association between the inferior frontal gyrus (IFG) and other brain regions results in better emotion regulation in reappraisal tasks. However, no study has explored the relationship between IFG-based resting-state functional connectivity (rsFC) and the dispositional use of reappraisal strategy. Therefore, the present study examined the potential associations between rsFC patterns of both left and right IFG and dispositional reappraisal use. One hundred healthy participants completed the Emotion Regulation Questionnaire $(E R Q)$ and underwent a resting-state functional magnetic resonance imaging (fMRI) acquisition. An approach of the seed-based rsFC analysis was recruited to estimate the functional connectivity maps of bilateral IFG with other brain regions, and the reappraisal scores from the $E R Q$ were then correlated with the functional maps. Our findings showed that IFG-based rsFC was positively correlated with dispositional reappraisal only in the range of 4 to 5.5 points [medium reappraisal group (MRG)]. Specifically, medium dispositional reappraisal was positively correlated with rsFC between left/right IFG and bilateral temporal gyrus. Besides, medium dispositional reappraisal was positively correlated with rsFC between left IFG and bilateral superior parietal lobe (SPL), middle cingulate cortex (MCC), and right insula, as well as between right IFG and dorsomedial prefrontal cortex (DMPFC) and anterior cingulate cortex (ACC). In conclusion, these results indicate that bilateral IFG plays an important role in the medium use of the reappraisal strategy.

Keywords: emotion regulation, inferior frontal gyrus, prediction, resting-state functional connectivity, medium reappraisal

\section{INTRODUCTION}

Effective emotion regulation is necessary for our daily social life. Essentially, various strategies can be employed to achieve successful emotion regulation, e.g., distraction, cognitive reappraisal, and expressive suppression (Webb et al., 2012; Morawetz et al., 2017b). Among these strategies, reappraisal, which entails the changing of the emotional value 
of stimuli that evokes emotions (Kanske et al., 2011; Webb et al., 2012), is the most frequently applied and studied strategy of emotion regulation (Wager et al., 2008; Kalisch, 2009; Ochsner et al., 2012; Buhle et al., 2014). Moreover, Gross and John (2003) developed a self-report Emotion Regulation Questionnaire (ERQ) to measure the dispositional use of two strategies, reappraisal (center on reinterpretation) and suppression. Assessment of this kind of personality habitude can reflect the utilization frequency of strategy, which may finally implicate the individual differences in abilities of emotion regulation. Also, more frequent use of reappraisal strategy has been demonstrated to be associated with better regulation of emotions, social interactions, and mental and physical health (Gross and John, 2003; Meyer et al., 2012; Hu et al., 2014; Picó-Pérez et al., 2018; Zaehringer et al., 2020).

The neural underpinnings related to reappraisal strategy have usually been evaluated by measurement of functional activation during experimental reappraisal tasks with functional magnetic resonance imaging (fMRI). The previous meta-analytic studies have shown that reappraisal recruits a widespread network that includes dorsomedial prefrontal cortex (DMPFC), dorsolateral prefrontal cortex (DLPFC)/superior frontal gyrus (SFG), ventrolateral prefrontal cortex (VLPFC)/inferior frontal gyrus (IFG), parietal lobes, temporal gyrus, and cingulate cortex (Phillips et al., 2008; Buhle et al., 2014; Kohn et al., 2014; Morawetz et al., 2017b). Importantly, the IFG/VLPFC is well known as a critical region for processes of selection and inhibition (Schulz et al., 2009; Kohn et al., 2014; Morawetz et al., 2017b), language (Ochsner et al., 2012; Messina et al., 2015), and social cognition (Kohn et al., 2014; Hartwigsen et al., 2019) in emotion regulation. In particular, the IFG has been observed with increased activation when multiple appropriate reinterpretations emerge and a choice must be made to achieve goal-directed behavior, as well as when required to inhibit goal-inappropriate reinterpretations (Morawetz et al., 2016; Braunstein et al., 2017).

Moreover, methods of IFG-based (with IFG serving as seed regions) functional connectivity have also been used to explore the neural correlations of reappraisal strategy in experimental settings. Morawetz et al. (2017a) defined left IFG as a seed region and examined effective connectivity between the seed region and the remaining brain regions, with reappraisal success scores as covariate. They have observed positive effective coupling between the left IFG and DLPFC, DMPFC, right middle temporal gyrus (MTG), and superior temporal gyrus (STG) during downregulation of emotion. Furthermore, in another study, Morawetz et al. (2016) found that the inhibitory effect on connectivity from IFG to DLPFC could facilitate successful reappraisal, deducing that the IFG may choose one from the many feasible goal-relevant reinterpretations actively maintained in the working memory (associated with DLPFC's increased activity), and suppress the DLPFC as soon as the selection process is finished. On the other hand, Wager et al. (2008) found that the right IFG could effectively predict reappraisal success with some cortical and subcortical regions as mediators, such as DMPFC, SFG, inferior temporal gyrus (ITG), and subgenual anterior cingulate cortex (ACC).
Recent evidence has shown that the formation of intrinsic resting-state functional architecture is influenced by repeated task-based co-activation within a network (Mackey et al., 2013; Guerra-Carrillo et al., 2014; Uchida et al., 2014), suggesting a close correspondence between task-specific brain activation and intrinsic brain connectivity, which is reflected by restingstate functional connectivity ( $\mathrm{rsFC}$ ). In essence, Smith et al. (2009) compared task-based activation networks derived from a large database of functional imaging studies with the covarying networks from 36 subjects' resting fMRI data, and found that these task-related networks closely matched the networks when at resting state. Intriguingly, another study using a sample of 4- to 18-year-old healthy participants found that task-related functional connectivity could even predict rsFC of up to 2 years after the initial experimental task (Gabard-Durnam et al., 2016). Thus, it seems possible that recurring activation caused by a specific task may share an association with resting-state connectivity pattern, and this may also apply to the emotion regulation domain with IFG-based functional connectivity during the reappraisal task. Besides, one study demonstrated that activation of IFG in reappraisal task is positively correlated with the frequency of dispositional reappraisal in daily life (Grecucci et al., 2013). Consequently, it can be assumed that the reappraisal task and dispositional reappraisal may share a similar IFG-based functional connectivity pattern. However, since no study has examined the association between dispositional use of reappraisal strategy and rsFC with IFG seed regions, it is uncertain whether IFG-based rsFC could also facilitate habitual reappraisal, thus resulting in better emotion regulation, although previous evidence indicates that both left and right IFG show associations with DMPFC and temporal gyrus during the performance of a reappraisal task. Nevertheless, it remains unknown whether the functional connectivity pattern of the left IFG seed concerning habitual reappraisal is the same as that of the right IFG seed.

Moreover, neural efficiency supposes that more adept individuals optimally use the functional connectivity to undertake minute neural processing and, hence, display diminished neural activity alongside the performance facilitation (Neubauer and Fink, 2009; Di Domenico et al., 2015; Curtin et al., 2019). It is anticipated that the higher the scores of dispositional reappraisal, the more frequent the use of reappraisal, and the better the ability of emotion regulation. Presumably, the neural efficiency may also be suitable for dispositional reappraisal, a daily used specific strategy of emotion regulation, with a changeable connectivity pattern along with a variation of reappraisal scores. However, there is no evidence supporting how the individual difference in frequency of reappraisal use may affect functional connectivity during the resting state. Considering the aforementioned close association between $\mathrm{rsFC}$ and task-related functional connectivity, we asked another question: Could it be possible that the IFG-based rsFC pattern vary with the level of frequency of dispositional reappraisal?

Therefore, in an endeavor to answer the questions raised, the present study applied seed-based rsFC analysis and prediction analysis with an aim of (1) examining whether IFG-based rsFC is related to individual dispositional use of reappraisal; (2) investigating whether habitual reappraisal related rsFC pattern of 
the left IFG is the same as that of the right IFG; (3) exploring whether IFG-based rsFC is specific to the frequency level of the use of dispositional reappraisal. Based on the limited evidence mentioned above, we hypothesized that dispositional use of reappraisal would be positively correlated with IFG-based rsFC and that the rsFC pattern of the left IFG and that of the right IFG would be very similar, with both showing associations with DMPFC and temporal gyrus. Moreover, according to the theory of neural efficiency (Neubauer and Fink, 2009; Di Domenico et al., 2015; Curtin et al., 2019), the individual differences in dispositional reappraisal may be associated with the difference in the IFG-based rsFC pattern, with a higher level of dispositional reappraisal corresponding to a less IFG-based rsFC.

\section{MATERIALS AND METHODS}

\section{Participants}

One hundred and seven $(N=107)$ healthy, right-handed adults (59 females, 17 to 26 years old, mean age $21.36 \pm 2.052$ years) participated in the experiment after giving their written informed consent. All participants reported no history of mental disorders, head injury, or cardiovascular diseases. The study protocol was approved by the Local Committee for the Protection of Human Subjects of the University of Electronic Science and Technology of China and was conducted according to the declaration of Helsinki.

\section{Behavioral Assessment}

All participants completed the ERQ (Gross and John, 2003) before scanning. The ERQ consisted of two subscales, cognitive reappraisal and expressive suppression. Ten items, with six for reappraisal and four for suppression, are included in the scale, whose choices ranged from strongly disagree (1) to strongly agree (7). In the current study, Cronbach's $\alpha$ coefficient of reappraisal subscale was 0.828 and that of suppression subscale was 0.601 . According to the scale used, four is the median score. In our interpretation, the individuals who scored less than four points do not frequently use either the reappraisal or suppression strategy. On the other hand, a score of more than four implies a frequent use of the aforementioned strategies. In our study, a very small number of participants $(n=7)$ recorded less than four points on the reappraisal subscale. Therefore, we found it appropriate to examine the intrinsic neural mechanisms of reappraisal strategy only among the individuals who frequently apply this strategy in their daily life. Hence, we excluded the data of $n=7$ participants with low frequent use of reappraisal strategy ( $<4$ points). Subsequently, we categorized the remaining $n=100$ participants into two groups, according to their reappraisal scores. Those who had a score of between 4 (median) and 5.5 were classified as moderate users of reappraisal strategy and thus put into the medium reappraisal group (MRG). Similarly, those with a score of between 5.5 and 7 were considered as highfrequency reappraisal strategy users and, therefore, categorized into high reappraisal group (HRG). Ultimately, all the remaining participants $(n=100)$ were assigned into either MRG $(n=80)$ or HRG $(n=20)$. In MRG, neither scores of reappraisal and suppression nor age showed significant gender differences (all $p>0.08$ ). This was the same case with HRG (all $p>0.4$ ) (Table 1). On the other hand, the reappraisal score was higher than the suppression score in both groups [MRG: $t_{(79)}=11.989$, $p<0.001$; HRG: $\left.t_{(19)}=11.995, p<0.001\right]$. Finally, to compare MRG with HRG, a subsample of 20 participants from MRG (named as sMRG) were selected, with similar age, gender, and suppression scores as HRG (all $p>0.4$ ) (Table 2).

\section{Image Acquisition and Data Analysis}

A 3.0-T GE Sigma scanner was used to collect resting-state fMRI images with a gradient echo planar imaging (EPI) sequence (TR, 2,000 ms; TE, $30 \mathrm{~ms}$; FA, 90 FOV, $240 \mathrm{~mm} \times 240 \mathrm{~mm}$; matrix size, $64 \times 64$; voxel size, $3.75 \mathrm{~mm} \times 3.75 \mathrm{~mm} \times 3 \mathrm{~mm}$; slices, 43). The T1-weighted structural image was acquired with a highresolution T1-weighted scan (TR, $5.96 \mathrm{~ms}$; TE, $1.96 \mathrm{~ms}$; FA, 9; FOV, $256 \mathrm{~mm} \times 256 \mathrm{~mm}$; matrix size, $256 \times 256$; voxel size, $1 \mathrm{~mm} \times 1 \mathrm{~mm} \times 1 \mathrm{~mm}$; number of slices, 176). Participants were instructed to rest with their eyes closed but not to fall asleep during the scan.

Resting-state fMRI data analysis was conducted using the data processing assistant for resting-state fMRI toolbox (DPARSF ${ }^{1}$ ) and statistical parametric mapping software $\left(\mathrm{SPM} 12^{2}\right)$. To keep magnetic field stabilization, the first five EPI volumes of the fMRI images were removed. Preprocessing consisted of the following steps: Slice timing correction, 3D motion correction, nuisance covariates regression (Friston-24 motion parameters; white matter, cerebrospinal fluid, and global signals), spatial

\footnotetext{
${ }^{1}$ http://rfmri.org/DPARSF

${ }^{2}$ http://www.fil.ion.ucl.ac.uk/spm
}

TABLE 1 | Demographic characteristics and behavioral assessment.

\begin{tabular}{|c|c|c|c|c|c|c|c|c|}
\hline & \multicolumn{4}{|c|}{ MRG } & \multicolumn{4}{|c|}{ HRG } \\
\hline Female & 44 & $21.20(2.11)$ & $4.93(0.35)$ & $3.40(0.91)$ & 12 & $21.25(1.82)$ & $6.04(0.44)$ & $3.52(0.89)$ \\
\hline Male & 36 & $21.58(2.09)$ & $4.92(0.33)$ & $3.76(0.94)$ & 8 & $21.25(2.05)$ & $6.21(0.54)$ & $3.50(1.16)$ \\
\hline
\end{tabular}

MRG, medium reappraisal group; HRG, high reappraisal group. 
normalization to the Montreal Neurological Institute (MNI) template and resampling to $3 \mathrm{~mm} \times 3 \mathrm{~mm} \times 3 \mathrm{~mm}$, removing the linear trends, temporal band-pass filtering $(0.01-0.1 \mathrm{~Hz})$, and spatial smoothing with a Gaussian kernel of full-width halfmaximum $6 \mathrm{~mm}$. The head motion exclusion was applied with translation not exceeding $3 \mathrm{~mm}$ and rotation not exceeding $3^{\circ}$, and mean FD_Jenkinson not exceeding 0.2 (Power et al., 2012; van Dijk et al., 2012). According to this threshold, no participant was excluded.

\section{Seed Definition}

Degree centrality refers to the number of brain connections from a voxel to others across the whole brain (Zuo et al., 2012; Yan et al., 2017; Lv et al., 2018). The measure of degree centrality has been widely performed to examine node characteristics of intrinsic connectivity networks, especially for the identification of functional hubs in functional connectivity analysis (Zuo et al., 2012). In the present study, a voxel-wise functional connectivity analysis was performed using DPARSFA, and the examination of degree centrality was recruited to identify functional hubs that were related to individual reappraisal scores. The correlation threshold was set at $r>0.25$ for the degree centrality calculation. Then, the resulting degree centrality was used to conduct a multiple regression analysis, with gender, age and suppression scores controlled as covariates of noninterest and reappraisal scores as a predictor of interest. For the exploratory purpose, we lowered the statistical threshold to $P_{\text {uncorrected }}<0.005$ with a cluster size $>50$ voxels. At this threshold, only two clusters emerged, the left and right IFG, whose degree centrality showed a positive correlation with reappraisal scores (Table 3 and Figure 1). Therefore, based on the results of the above voxel-wise

TABLE 2 | Comparison of behavioral assessment between HRG and SMRG.

\begin{tabular}{|c|c|c|c|c|}
\hline & Gender & Age & Reappraisal & Suppression \\
\hline & Female/Male & M (SD) & M (SD) & M (SD) \\
\hline HRG & $12 / 8$ & $21.25(1.86)$ & $6.11(0.48)$ & $3.51(0.98)$ \\
\hline sMRG & $12 / 8$ & $21.70(1.95)$ & $4.90(0.29)$ & $3.43(0.85)$ \\
\hline$p$ & 1.000 & 0.460 & $<0.001$ & 0.764 \\
\hline
\end{tabular}

HRG, high reappraisal group; sMRG, subsample of medium reappraisal group.

TABLE 3 | Results of voxel-wise functional connectivity.

\begin{tabular}{|c|c|c|c|c|c|c|}
\hline \multirow[t]{2}{*}{ Region } & \multirow[t]{2}{*}{$\mathbf{H}$} & \multirow[t]{2}{*}{$\mathbf{K}$} & \multirow[t]{2}{*}{$\mathbf{T}$} & \multicolumn{3}{|c|}{ MNI coordinates } \\
\hline & & & & $x$ & $y$ & $z$ \\
\hline \multirow[t]{3}{*}{ Inferior frontal gyrus } & $\mathrm{L}$ & 106 & 4.33 & -54 & 6 & 15 \\
\hline & & & 3.85 & -45 & 9 & 18 \\
\hline & & & 3.27 & -51 & 12 & 3 \\
\hline \multirow[t]{3}{*}{ Inferior frontal gyrus } & $R$ & 69 & 3.85 & 45 & 12 & 9 \\
\hline & & & 3.46 & 48 & 3 & 15 \\
\hline & & & 3.42 & 54 & 12 & 15 \\
\hline
\end{tabular}

$H$, hemisphere; $L$, left; $R$, right; $K$, cluster size in number of activated voxels; $T, T$ value; MNI, the Montreal Neurological Institute coordinates.

Statistical threshold of $P_{\text {uncorrected }}<0.005$ was used for cluster correcting.

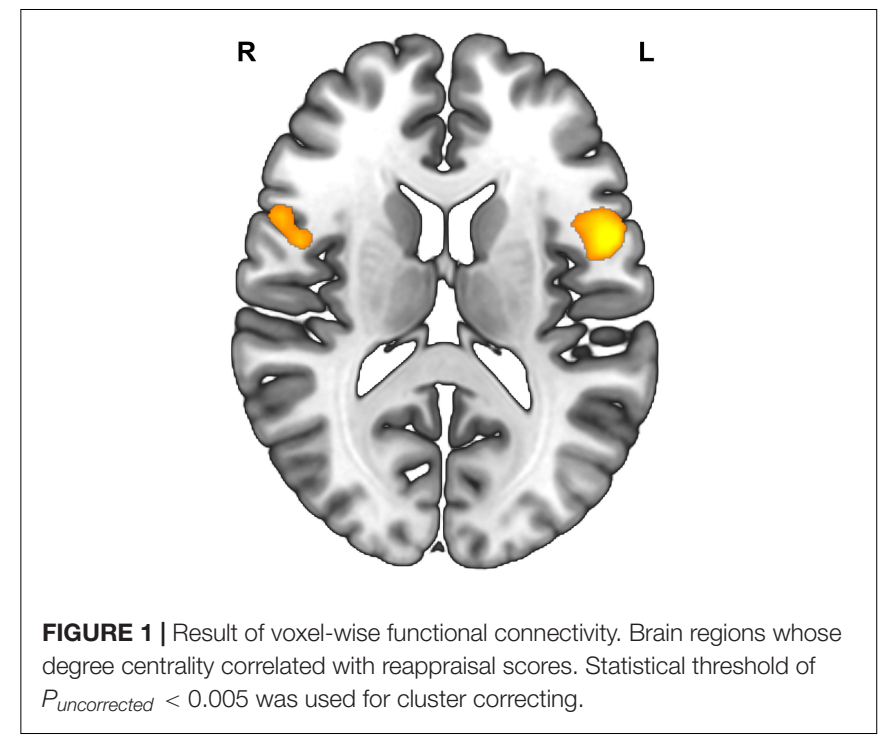

functional connectivity analysis, left IFG (-48 9 6) and right IFG (54 12 18) were defined as seed regions for further seed-based rsFC analysis. The two seed regions were separately built as a 6$\mathrm{mm}$ radius sphere centered around the peak activation using the Marsbar toolbox ${ }^{3}$.

\section{Seed-Based Voxel-Wise rsFC Analysis}

After seeds extraction, seed-based voxel-wise rsFC analyses were performed to explore brain regions connected with left/right IFG, and the connectivity correlating with individual reappraisal scores. Firstly, time series of all voxels located within these two seed regions were abstracted and averaged, respectively. Secondly, a Pearson correlation was conducted between each seed region's time series and those of all other brain voxels of each participant. Thereafter, the resulting correlation coefficients were transformed into Fisher's $z$ scores, representing the rsFC for each connection of each participant. Subsequently, multiple regression models were performed with reappraisal scores as a predictor of interest, and the effect of gender, age, and suppression scores simultaneously eliminated. All activations were applied at the whole-brain level with a statistical significance of false discovery rate $P_{F D R}<0.05$ and a cluster extent $>50$ voxels. Besides, multiple regression models were also performed with suppression scores as a predictor of interest, suggested by PicóPérez et al. (2018) in their similar study on the association between dispositional use of emotional regulation strategies and rsFC, but with the amygdala as seed regions. However, we did not observe any significant activation when we used the suppression scores as a predictor of interest at the same threshold of $P_{F D R}<0.05$. Consequently, the suppression strategy was not included in the result.

In addition, we further extracted the rsFC strength value of each region of interest (ROI), which amounted to a sphere of $6-\mathrm{mm}$ radius centered around the peak of activation using Marsbar toolbox (see text footnote 3). Then, the partial

${ }^{3}$ http://marsbar.sourceforge.net 
correlation analyses were performed between connectivity strength and reappraisal scores after controlling for gender, age, and suppression scores.

Initially, we tried to investigate the potential association between IFG-based rsFC and reappraisal scores across the entire range observed in the sample of $n=100$. Unfortunately, we did not find any significant activation at the threshold of $P_{F D R}<0.05$. The previous literature, especially the theory of neural efficiency, may provide a reasonable conjecture that individuals with a higher level of frequency of dispositional reappraisal use may show less connectivity between IFG and the remaining regions. Hence, we considered that, probably, the high-frequency level of dispositional use of reappraisal affects the association between IFG-based rsFC and reappraisal scores. Therefore, as reported before, the 100 participants were allocated into two groups (MRG and HRG) according to the range of reappraisal scores.

\section{Prediction Analysis Using Cross-Validation}

To test whether the observed functional brain features in MRG could reliably predict reappraisal scores of new individuals, internal cross-validation analyses were performed using the Pattern Recognition for Neuroimaging Toolbox (PRoNTo v2.1 ${ }^{4}$ ). The input vectors were mean-centered using the training data (Xie et al., 2020), while the effect of covariates of noninterest (gender, age, and suppression scores) was regressed out. The predictive power was assessed by calculating Pearson's correlation coefficients between the predicted and actual reappraisal scores. Additionally, the statistical significance of the correlation was determined by 5,000 times of permutation testing without replacement.

In order to examine whether the reappraisal related IFG-based rsFC pattern in MRG is the same as that in HRG, internal crossvalidation analyses were conducted using the reappraisal related rsFC of sMRG to predict the reappraisal scores of HRG.

\section{RESULTS}

\section{Functional Connectivity Analysis}

In MRG, with the left IFG as a seed region, reappraisal scores were positively correlated with rsFC between left IFG and most bilateral regions consisting of STG/MTG/ITG, superior parietal lobe (SPL), middle cingulate cortex (MCC), postcentral/precentral gyrus, rolandic operculum, cerebellum, and fusiform gyrus; and between left IFG and some left regions including inferior parietal lobe (IPL), supplementary motor area (SMA), precuneus, and occipital gyrus, as well as between left IFG and right insula (Table 4 and Figure 2A). However, there was no significant activation at the same threshold level in HRG.

Functional connectivity analysis with right IFG as a seed region in MRG showed that reappraisal scores were positively correlated with rsFC between right IFG and brain areas such as the bilateral medial SFG, bilateral STG/MTG/ITG, bilateral precuneus, bilateral postcentral, bilateral occipital gyrus, bilateral fusiform, left ACC, left SFG, left insula, and right supramarginal

${ }^{4}$ www.mlnl.cs.ucl.ac.uk/pronto/ gyrus (Table 4 and Figure 3A). Although in HRG, there were still no significant activation yielded at the same threshold level.

\section{Association of rsFC Strength With Reappraisal}

When considering the left IFG as a seed region in MRG, the following ROIs showed significant correlation between strength values and reappraisal scores: left SPL $(-21-5748), r=0.432$, $p<0.001$; right SPL $(24-6051), r=0.388, p<0.001$; right insula $(42-126), r=0.431, p<0.001$; left STG $(-57-2712), r=0.476$, $p<0.001$; right STG $(54-216), r=0.450, p<0.001$; right MCC $(9-1542), r=0.378, p=0.001$ (Figure 2B).

On the other hand, when the right IFG is considered as a seed region in MRG, the following ROIs showed significant correlation between strength and reappraisal scores: DMPFC (rostral cluster) $(-65118), r=0.506, p<0.001$; DMPFC (caudal cluster) ( -63651$), r=0.442, p<0.001$; left ACC (-6 48 12), $r=0.442, p<0.001$; left MTG $(-48-576), r=0.395, p<0.001$ right MTG $(42-603), r=0.428, p<0.001$; right STG $(57-42$ 21), $r=0.378, p=0.001$ (Figure 3B).

\section{Prediction Analysis}

In MRG, the left IFG seed-region-related rsFC could effectively predict for individual reappraisal scores $(r=0.370, p=0.002)$ (Figure 4A), and this was also true for the right IFG seed region $(r=0.330, p=0.004)$ (Figure 4B). However, there was no significant predictive power for individual reappraisal scores of HRG with left IFG or right IFG seed-region-related rsFC in sMRG (left IFG: $r=-0.380, p=0.636$, Figure 5A; right IFG: $r=-0.590, p=0.856$, Figure 5B).

\section{DISCUSSION}

Previous studies have emphasized the key functional role of IFG in collaboration with other regions in emotional regulation taskbased functional connectivity, while the present study constitutes the first investigation into the associations between rsFC patterns of bilateral IFG and the dispositional use of reappraisal. Interestingly, we observed that medium dispositional use of reappraisal was positively related to IFG-based rsFC. Specifically, the medium habitual use of reappraisal was associated with a significant positive coupling between: (1) bilateral IFG and temporal gyrus; (2) left IFG and bilateral SPL, MCC, left IPL, and right insula; and (3) right IFG and DMPFC/ACC. However, no significant correlation emerged between left or right IFG-related rsFC and the high dispositional reappraisal use. The predictive analyses also showed that both left and right IFG-related rsFC could effectively and separately predict for individual reappraisal scores in MRG. However, IFG-related rsFC of sMRG had no significant predictive power for reappraisal scores of HRG.

In line with our hypotheses, the associations of both left and right IFG with temporal gyrus were linked to the habitual use of reappraisal. Particularly, using the reappraisal strategy altered the emotional value of stimuli by manipulating the conceptual knowledge and creating opposite interpretations. This suggests that the involvement of the semantic process is a core part 
TABLE 4 | Functional connectivity results.

\begin{tabular}{|c|c|c|c|c|c|c|}
\hline \multirow[t]{2}{*}{ Region } & \multirow[t]{2}{*}{$\mathbf{H}$} & \multirow[t]{2}{*}{$\mathbf{K}$} & \multirow[t]{2}{*}{$\mathbf{T}$} & \multicolumn{3}{|c|}{ MNI coordinates } \\
\hline & & & & $x$ & $y$ & $Z$ \\
\hline \multicolumn{7}{|l|}{ Left IFG as a seed } \\
\hline \multirow[t]{2}{*}{ Superior temporal gyrus/middle temporal gyrus/rolandic operculum } & $\mathrm{L}$ & 320 & 5.50 & -57 & -30 & 3 \\
\hline & & & 5.26 & -57 & -27 & 12 \\
\hline Postcentral gyrus/precentral gyrus/superior parietal lobe/inferior parietal & $\mathrm{L}$ & 949 & 5.09 & -21 & -36 & 66 \\
\hline lobe/middle cingulate cortex/supplementary motor area/precuneus & & & 4.64 & -21 & -51 & 51 \\
\hline \multirow[t]{3}{*}{ Cerebellum/fusiform/inferior temporal gyrus } & $\mathrm{R}$ & 314 & 5.01 & 24 & -54 & -18 \\
\hline & & & 4.59 & 39 & -54 & -15 \\
\hline & & & 4.46 & 48 & -48 & -21 \\
\hline \multirow[t]{3}{*}{ Superior temporal gyrus/middle temporal gyrus/rolandic operculum/insula } & $\mathrm{R}$ & 562 & 4.81 & 72 & -30 & 0 \\
\hline & & & 4.57 & 39 & -12 & 6 \\
\hline & & & 4.49 & 63 & 3 & 6 \\
\hline Postcentral gyrus/precentral gyrus/superior parietal lobe & $\mathrm{R}$ & 320 & 4.61 & 21 & -36 & 66 \\
\hline \multirow[t]{2}{*}{ Cerebellum/fusiform/inferior temporal gyrus } & $\mathrm{L}$ & 385 & 4.68 & -42 & -57 & -24 \\
\hline & & & 4.42 & -39 & -66 & -18 \\
\hline Middle occipital gyrus & $\mathrm{L}$ & 128 & 4.65 & -30 & -78 & 12 \\
\hline \multicolumn{7}{|l|}{ Right IFG as a seed } \\
\hline Superior medial frontal gyrus/anterior cingulate cortex & $\mathrm{L}$ & 294 & 5.45 & -6 & 51 & 18 \\
\hline \multirow{2}{*}{$\begin{array}{l}\text { Cerebellum/fusiform/superior occipital gyrus/hippocampus/middle temporal } \\
\text { gyrus/inferior temporal gyrus }\end{array}$} & $\mathrm{L}$ & 1560 & 5.00 & -36 & -33 & -27 \\
\hline & & & 4.71 & -30 & -9 & -21 \\
\hline Superior temporal gyrus/middle temporal gyrus & $\mathrm{R}$ & 136 & 4.19 & 57 & -42 & 21 \\
\hline \multirow[t]{2}{*}{ Superior temporal gyrus/middle temporal gyrus } & $\mathrm{L}$ & 299 & 4.43 & -45 & -45 & 12 \\
\hline & & & 3.88 & -48 & -57 & 6 \\
\hline Precuneus & $\mathrm{L}$ & 111 & 4.40 & -9 & -54 & 45 \\
\hline \multirow[t]{2}{*}{ Superior medial frontal gyrus/superior frontal gyrus } & $\mathrm{L}$ & 158 & 4.35 & -6 & 36 & 51 \\
\hline & $\mathrm{R}$ & & 3.87 & 3 & 39 & 48 \\
\hline Superior occipital gyrus & $\mathrm{R}$ & 152 & 4.09 & 15 & -90 & 21 \\
\hline \multirow[t]{2}{*}{ Middle temporal gyrus/insula } & $\mathrm{L}$ & 114 & 4.11 & -60 & -15 & -9 \\
\hline & & & 4.07 & -45 & -9 & 3 \\
\hline \multirow[t]{2}{*}{ Postcentral gyrus/Precuneus } & $\mathrm{R}$ & 101 & 3.92 & 27 & -39 & 51 \\
\hline & & & 3.47 & 12 & -54 & 45 \\
\hline \multirow[t]{2}{*}{ Supramarginal gyrus/postcentral gyrus } & $\mathrm{L}$ & 144 & 3.66 & -54 & -27 & 30 \\
\hline & & & 3.61 & -54 & -12 & 24 \\
\hline
\end{tabular}

$H$, hemisphere; L, left; $R$, right; $K$, cluster size in number of activated voxels; T, T value; MNI, the Montreal Neurological Institute coordinates.

Statistical threshold of false discovery rate $P_{F D R}<0.05$ was used for cluster correcting.

of emotion regulation. Evidently, the semantic system plays a critical role in the storage and controlled retrieval of conceptual knowledge (Binder et al., 2009), contributing to representations of relevant emotional information from emotional experiences (Neumann and Lozo, 2012). Specifically, the temporal gyrus, which has often been observed with enhanced activation in reappraisal task (Goldin et al., 2008; Kanske et al., 2011; Dörfel et al., 2014), is usually considered as part of semantic system (Binder et al., 2009) and plays a role in both the storage and the strategic retrieval of semantic knowledge (Davey et al., 2016). Recent evidence on functional connectivity between IFG and MTG at both task (Zhang et al., 2019) and resting-state contexts (Kohn et al., 2014; Davey et al., 2016) indicates that the cooperation between IFG and MTG makes strategic access of semantic information possible. Similarly, the present study also revealed strong functional connectivity between IFG and temporal gyrus, alongside a positive correlation with medium habitual use of reappraisal, perhaps supporting the capacity to potentially engage and sustain semantic retrieval, in line with goal-driven control of subjective emotional feelings.

Inconsistent with our preliminary expectation, only the right IFG displayed an association with DMPFC. The DMPFC has generally been proved to be involved in semantic and selfreflective processes (Olsson and Ochsner, 2008; Binder et al., 2009), and has repeatedly been observed to be significantly activated in the reappraisal tasks (Kanske et al., 2011; Buhle et al., 2014; Morawetz et al., 2017a, 2016). In particular, DMPFC is implicated with the elaboration of the affective meaning of stimuli and representation of value information concerning mental states (Ochsner et al., 2012; Dixon et al., 2017). Thus, the right IFG, with a close connection with DMPFC, may facilitate the evaluation of the changing mental states, in relation to outcomes 
A

$\mathbf{L}$
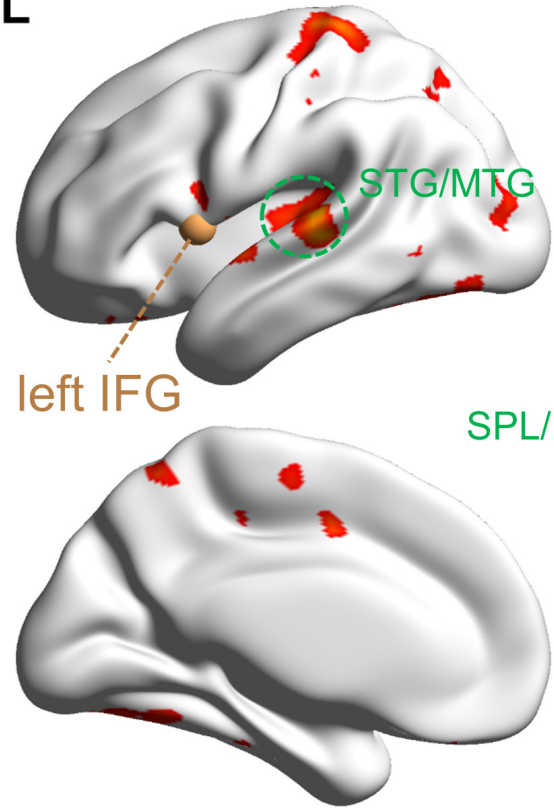

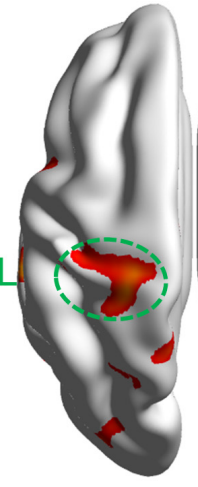

3

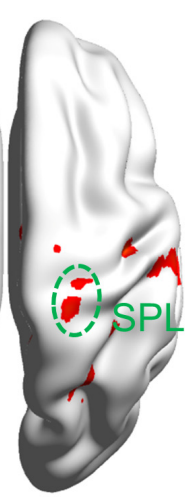

(T)

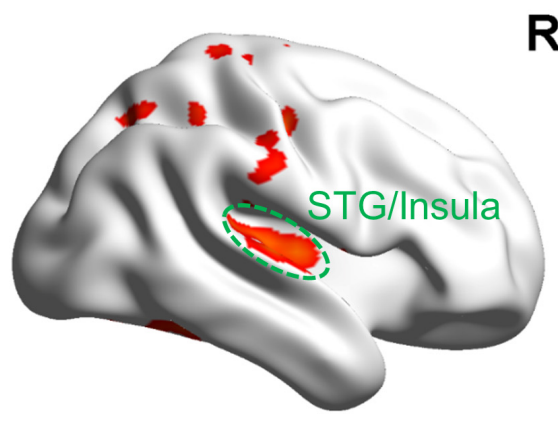

$\mathbf{R}$

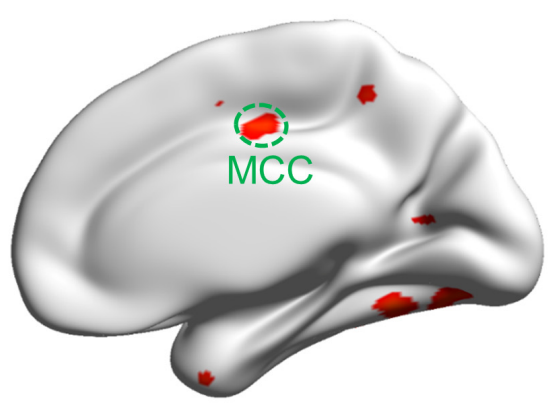

B
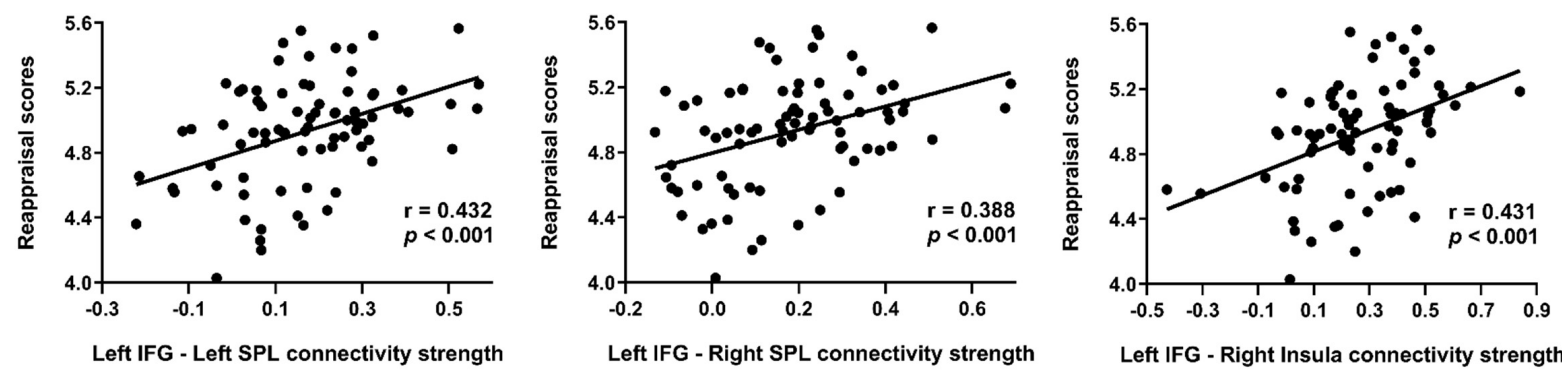

Left IFG - Right SPL connectivity strength

Left IFG - Right Insula connectivity strength

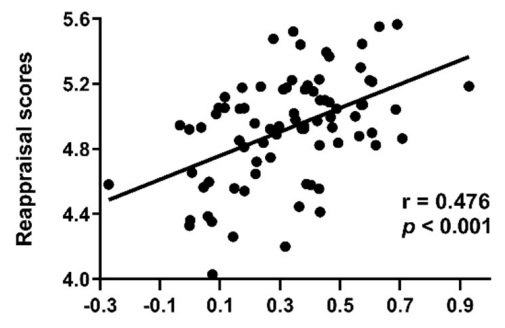

Left IFG - Left STG connectivity strength

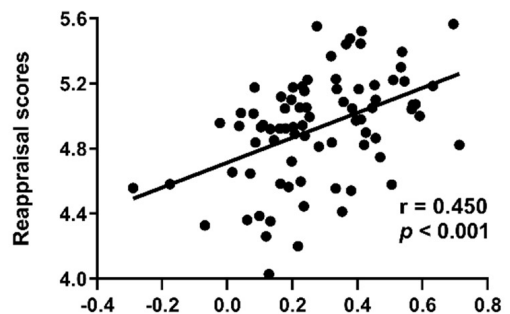

Left IFG - Right STG connectivity strength

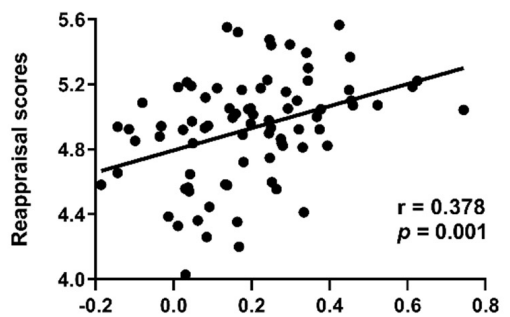

Left IFG - Right MCC connectivity strength

FIGURE 2 | Results of the left IFG-based rsFC related with dispositional use of reappraisal in MRG. (A) Brain regions whose rsFC correlated with reappraisal scores. The brown sphere represented the seed region, and the green circles were drawn to display the ROls. (B) Partial regression scatter plots depicted the correlation between functional connectivity strength and reappraisal scores. Statistical threshold of false discovery rate $P_{F D R}<0.05$ was used for cluster correcting.

of appropriate or inappropriate interpretations of emotional stimuli. Besides, with a correlation with medium habitual reappraisal use, right IFG-based rsFC also showed a strong link with ACC. Recent evidence suggests that ACC constitutes a core part of the neural circuitry of valuation (Amemori and Graybiel, 2012; Bartra et al., 2013; Clithero and Rangel, 2013), playing an important role in evaluating interoceptive signals based on self-referential and conceptual emotion knowledge (Dixon et al., 2017). The evaluation role of ACC may thus facilitate a better understanding of subjective emotional feelings, by assigning conceptual meaning to these bodily sensations. Notably, through interaction with ACC, DMPFC contributes to 
A
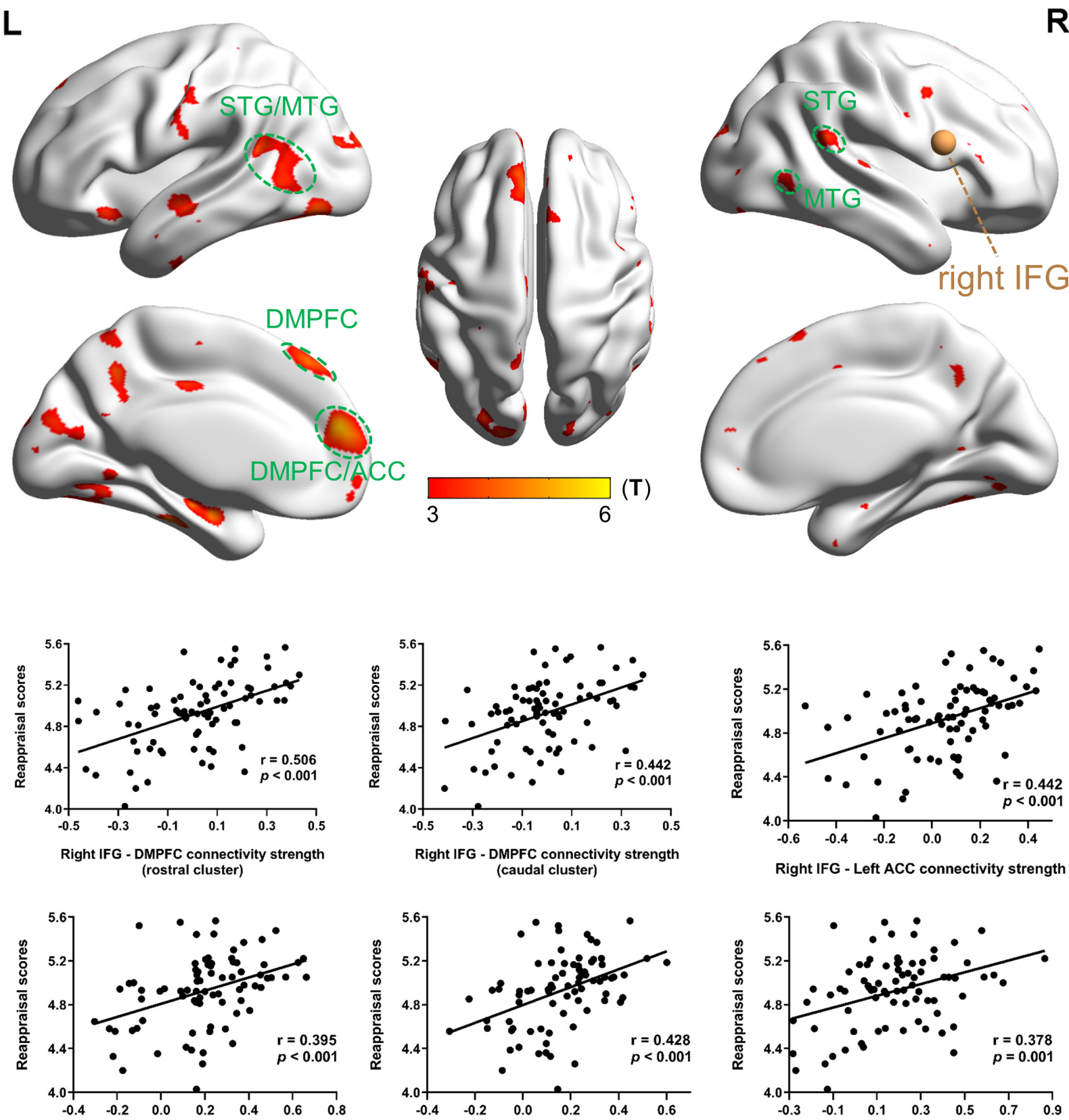

Right IFG - Left MTG connectivity strength

Right IFG - Right MTG connectivity strength

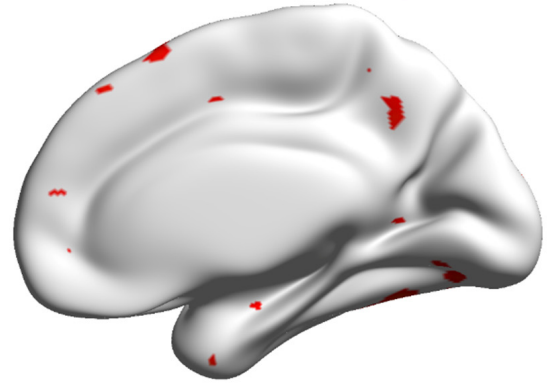

Right IFG - Left ACC connectivity strength

Right IFG - Right STG connectivity strength

FIGURE 3 | Results of the right IFG-based rsFC related with dispositional use of reappraisal in MRG. (A) Brain regions whose rsFC correlated with reappraisal scores. The brown sphere represented the seed region, and the green circles were drawn to display the ROls. (B) Partial regression scatter plots depicted the correlation between functional connectivity strength and reappraisal scores. Statistical threshold of false discovery rate $P_{F D R}<0.05$ was used for cluster correcting.

the maintenance of mental representations of an individual's feelings active in affective working memory (Lane et al., 2015) and may subsequently transfer these internal state information to IFG via a feed-forward mechanism (Phillips et al., 2008). Therefore, it is highly plausible that the right IFG (roles in selecting appropriate or inhibiting inappropriate interpretations from semantic memory) strongly connects with DMPFC and ACC (roles in perceiving and evaluating subjective emotional feelings), exhibiting correspondingly more frequent reappraisal skill, to achieve goal-directed emotional states.

Furthermore, as indicated above, we also observed a positive correlation between medium dispositional use of reappraisal 
Predicting reappraisal scores using reappraisal scores related left IFG_rsFC
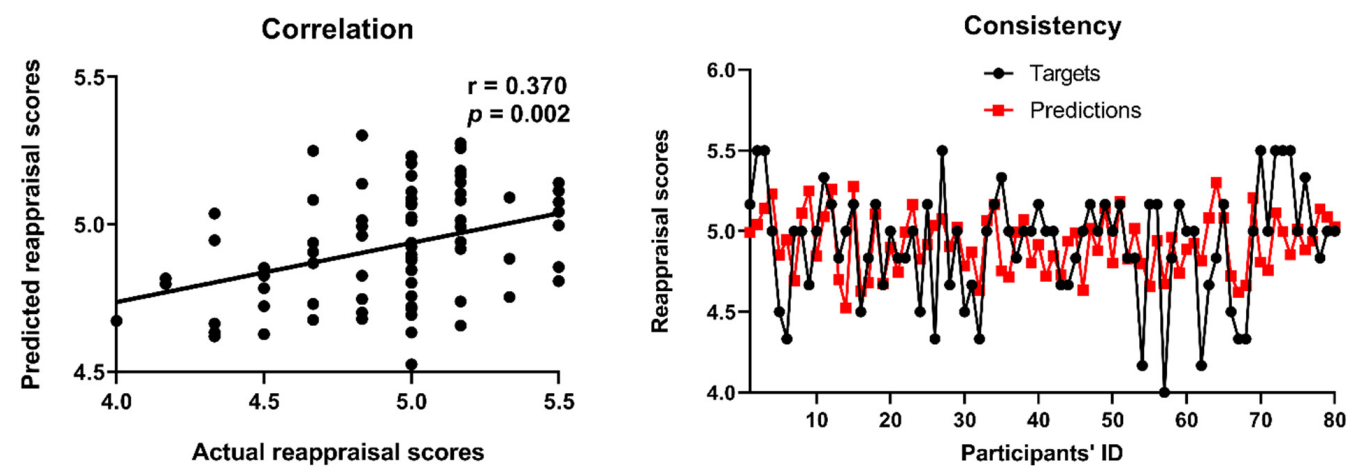

Predicting reappraisal scores using reappraisal scores related right IFG_rsFC
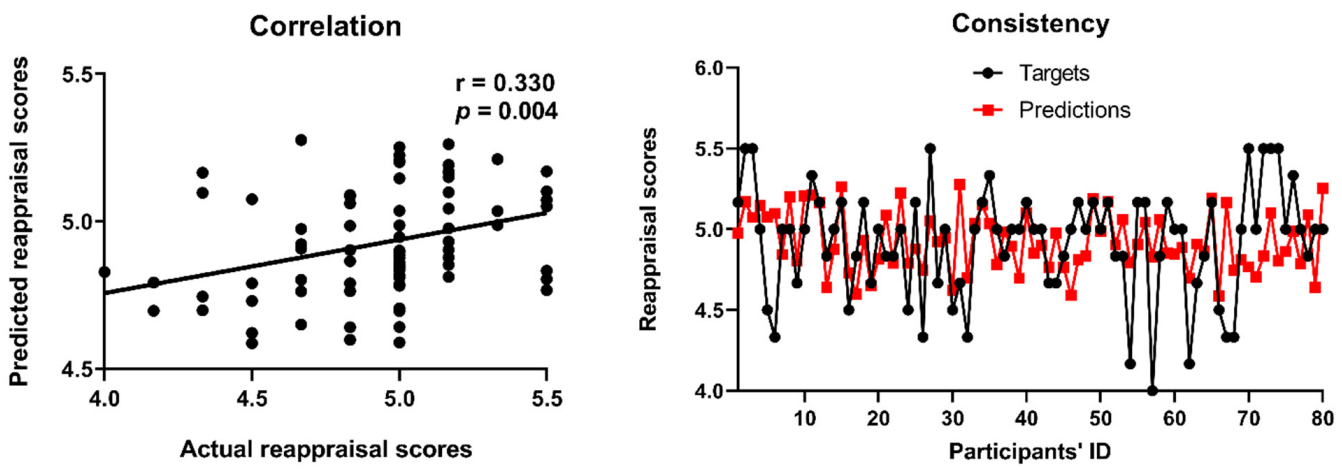

FIGURE 4 | Results of prediction in MRG. Severally using (A) the left and (B) the right IFG-based rsFC to predict reappraisal scores. The scatter plots and line charts [in both panels $(\mathbf{A}, \mathbf{B})$ ] described a significant correlation and consistency between actual and predicted reappraisal scores, respectively.

and connectivity of left IFG with bilateral SPL, MCC, left IPL, and right insula during resting state. Consistent with the previous findings on the activation of parietal lobes in reappraisal task (Buhle et al., 2014; Morawetz et al., 2017b), our study also found an association between habitual use of reappraisal and co-activation of left IFG and parietal lobes (including SPL and IPL), which usually engage in the attention control process (Luks et al., 2007; Hutchinson et al., 2014; Salo et al., 2017; Sang et al., 2017; Wang et al., 2020). More importantly, it is documented that the parietal lobes and prefrontal gyrus engage in cognitive control by exerting influence on the temporal regions to change the semantic and perceptual representations, so as to facilitate the selection of appropriate behaviors, and the inhibition of maladaptive habitual actions (Buhle et al., 2014; Dixon, 2015). Similarly, MCC has also been found to be strongly involved in the allocation of attention to emotional information and action monitoring (McRae et al., 2008; Kragel et al., 2018). Indeed, the cognitive function of MCC in performance monitoring may help guide the changing emotional responses through reappraisal strategy in an intended way (Ochsner et al., 2012). Moreover, MCC also combines with the insula, which contains bodily information and sensations (including interoceptive representation of emotions) (Craig, 2009; Lane et al., 2015), to project the affective information to the neighboring IFG/VLPFC (Craig, 2009; Kohn et al., 2014), indicating a motivation to IFG to select an appropriate response in the final stage. During this process, IFG may work in concert with the parietal lobes and temporal gyrus to focus attention on the subjective feelings and select appropriate interpretation to obtain a desirable emotional state.

As initially anticipated, we did not observe a significant correlation between left or right IFG-related rsFC and the high dispositional reappraisal use. More so, the IFG-related rsFC pattern of MRG could not effectively predict for reappraisal scores of HRG. This supports the neural efficiency view, that the more adept the skill, the lesser the neural connectivity, but the more enhanced the performance becomes (Neubauer and Fink, 2009; Di Domenico et al., 2015; Curtin et al., 2019). However, there is a conspicuous lack of evidence on trait emotion regulation with reappraisal disposition to support our findings. Nevertheless, some promising evidence from cognitive training provides a potential explanation. For example, Vartanian et al. (2013) proved that working memory training can augment performance on divergent thinking task 


\section{A Predicting High-reappraisal scores using Medium-reappraisal scores related left IFG_rsFC}
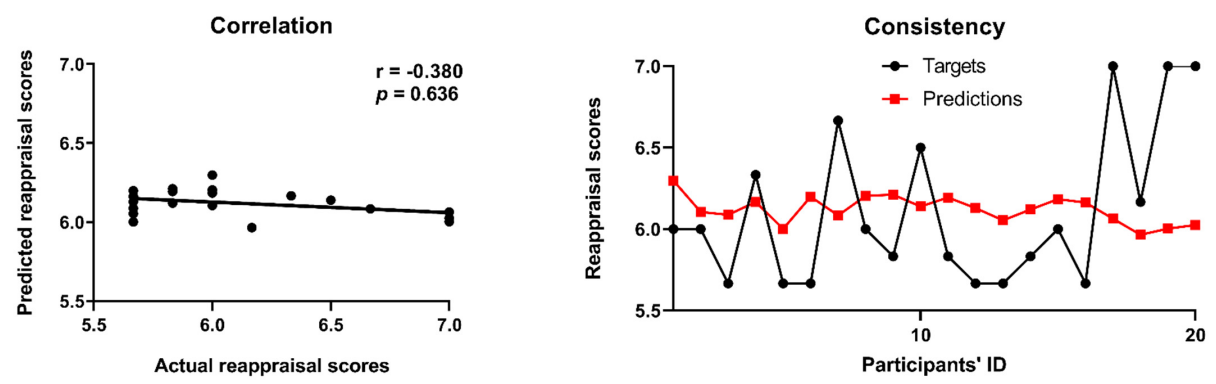

Predicting High-reappraisal scores using Medium-reappraisal scores related right IFG_rsFC
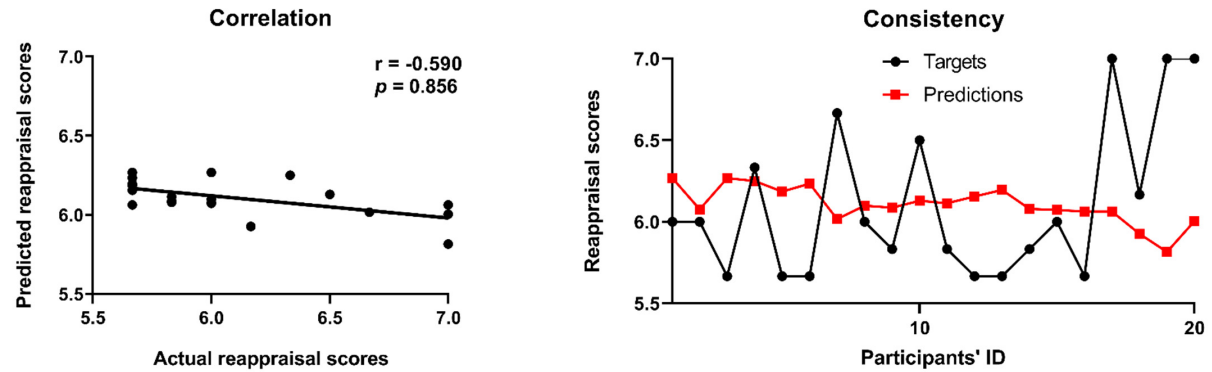

FIGURE $\mathbf{5}$ | Results of prediction between HRG and sMRG. We separately used (A) the left and (B) the right IFG-based rsFC of sMRG to predict the reappraisal scores of HRG. Scatter plots and line charts indicated non-significant correlation and consistency between actual and predicted reappraisal scores, respectively.

and lead to lower activation in the prefrontal gyrus (VLPFC and DLPFC) in adult participants, while Motes et al. (2018) found that cognitive training results in a faster processing speed along with reduced activation in the prefrontal gyrus in elderly participants. Therefore, it is possible that individuals with highfrequency daily use of reappraisal strategy, possess a more adept skill of emotion regulation, and recruit less functional connectivity between IFG and other regions in the resting state. On the other hand, the limited sample size in HRG may also be a potential reason to explain the non-significant results of both the association between rsFC and reappraisal scores, and the correlation in the internal cross-validation analysis. If so, future study should bring into consideration the sample size factor to ascertain whether the IFG-based rsFC pattern is linked to the variation of frequency level of dispositional use of reappraisal.

Overall, previous studies have emphasized the important role of the IFG in cooperation with other brain regions in task-based functional connectivity, in the selection and inhibition processes of emotional regulation. The present study expands on these findings by explicitly investigating how patterns of functional connectivity between IFG and other brain regions change during resting state, and how these changes may be linked to individuals' habitual reappraisal use. Specifically, besides coupling with temporal gyrus in the function of general semantic control, we found that the left IFG, along with SPL, MCC, left IPL and right insula, predominantly engages in monitoring the emotional performance (cognitive control of emotion), while the right IFG, coupling with DMPFC and ACC, predominantly engages in representation of mental states (evaluation of emotion).

Despite the important contributions of our study, there are several limitations needed to be noted. Firstly, as mentioned above, the sample size of HRG is relatively smaller than that of the MRG. Possibly, there may be some other potential intrinsic functional connectivity patterns recruited by individuals with higher emotion regulation capacity. To fully understand the neural substrates of emotion regulation, more participants with a higher ability of emotion regulation need to be included in future studies. Secondly, the present study only recruited healthy participants. Although the neural association observed in these participants may provide potential neural evidence for clinical practice, the dispositional reappraisal use may show a different association with the resting-state networks in the context of emotional dysregulation. Therefore, future studies should consider a comparative analysis of the relevance of habitual strategy use and regulation networks, between patients with emotional disorders and healthy populations, to improve the clinical understanding and intervention. Finally, although we have not observed a significant association between IFG-based rsFC and suppression scores, which is also a personality trait of emotion regulation, it may exist in other potential functional hubs and neural substrates relevant to the dispositional use of the suppression strategy. Thus, future studies should explore the potential neural mechanism underlying trait suppression in both healthy and clinical populations. 
In conclusion, the present study investigated the intrinsic neural underpinnings of dispositional reappraisal employing the IFG-based function connectivity approach during resting state. Our findings demonstrate that the medium dispositional reappraisal use relies on the cooperation of the functional hubs of the bilateral IFG and other regions within the emotion regulation cortex. These findings may explain how individuals cope with emotional events in daily life, as well as applied in clinical intervention for emotion-regulation-related disorders.

\section{DATA AVAILABILITY STATEMENT}

The original contributions presented in the study are included in the article, further inquiries can be directed to the corresponding authors.

\section{ETHICS STATEMENT}

The studies involving human participants were reviewed and approved by the Local Committee for the Protection of Human

\section{REFERENCES}

Amemori, K. I., and Graybiel, A. M. (2012). Localized microstimulation of primate pregenual cingulate cortex induces negative decision-making. Nat. Neurosci. 15, 776-785. doi: 10.1038/nn.3088

Bartra, O., McGuire, J. T., and Kable, J. W. (2013). The valuation system: a coordinate-based meta-analysis of BOLD fMRI experiments examining neural correlates of subjective value. Neuroimage 76, 412-427. doi: 10.1016/j. neuroimage.2013.02.063

Binder, J. R., Desai, R. H., Graves, W. W., and Conant, L. L. (2009). Where is the semantic system? A critical review and meta-analysis of 120 functional neuroimaging studies. Cereb. Cortex 19, 2767-2796. doi: 10.1093/cercor/ bhp055

Braunstein, L. M., Gross, J. J., and Ochsner, K. N. (2017). Explicit and implicit emotion regulation: a multi-level framework. Soc. Cogn. Affect. Neurosci. 12, 1545-1557. doi: 10.1093/scan/nsx096

Buhle, J. T., Silvers, J. A., Wage, T. D., Lopez, R., Onyemekwu, C., Kober, H., et al. (2014). Cognitive reappraisal of emotion: a meta-analysis of human neuroimaging studies. Cereb. Cortex 24, 2981-2990. doi: 10.1093/cercor/bht154

Clithero, J. A., and Rangel, A. (2013). Informatic parcellation of the network involved in the computation of subjective value. Soc. Cogn. Affect. Neurosci. 9, 1289-1302. doi: 10.1093/scan/nst106

Craig, A. D. (2009). How do you feel - now? The anterior insula and human awareness. Nat. Rev. Neurosci. 10, 59-70. doi: 10.1038/nrn2555

Curtin, A., Ayaz, H., Tang, Y., Sun, J., Wang, J., and Tong, S. (2019). Enhancing neural efficiency of cognitive processing speed via training and neurostimulation: an fNIRS and TMS study. Neuroimage 198, 73-82. doi: 10. 1016/j.neuroimage.2019.05.020

Davey, J., Thompson, H. E., Hallam, G., Karapanagiotidis, T., Murphy, C., De Caso, I., et al. (2016). Exploring the role of the posterior middle temporal gyrus in semantic cognition: integration of anterior temporal lobe with executive processes. Neuroimage 137, 165-177. doi: 10.1016/j.neuroimage.2016.05.051

Di Domenico, S. I., Rodrigo, A. H., Ayaz, H., Fournier, M. A., and Ruocco, A. C. (2015). Decision-making conflict and the neural efficiency hypothesis of intelligence: a functional near-infrared spectroscopy investigation. Neuroimage 109, 307-317. doi: 10.1016/j.neuroimage.2015.01.039

Dixon, M. L. (2015). Cognitive control, emotional value, and the lateral prefrontal cortex. Front. Psychol. 6:758. doi: 10.3389/fpsyg.2015.00758

Dixon, M. L., Thiruchselvam, R., Todd, R., and Christoff, K. (2017). Emotion and the prefrontal cortex: an integrative review. Psychol. Bull. 143, 1033-1081. doi: $10.1037 /$ bul0000096
Subjects of the University of Electronic Science and Technology of China. Written informed consent to participate in this study was provided by the participants.

\section{AUTHOR CONTRIBUTIONS}

WL, JZ, ZJ, and LL conceived and designed the experiments. WL performed the experiments. WL and KX analyzed the data. WL wrote the main manuscript text. RN revised the manuscript. All authors reviewed the manuscript.

\section{FUNDING}

This work was supported by grants from NSFC (61773092, 61673087, and 61773096), by the Sichuan Province Science and Technology Support Program (2020YFS0230), by the Higher Education Discipline Innovation Project (111 project, grant number B12027), and by the Fundamental Research Funds for the Central Universities.

Dörfel, D., Lamke, J. P., Hummel, F., Wagner, U., Erk, S., and Walter, H. (2014). Common and differential neural networks of emotion regulation by detachment, reinterpretation, distraction, and expressive suppression: a comparative fMRI investigation. Neuroimage 101, 298-309. doi: 10.1016/j. neuroimage.2014.06.051

Gabard-Durnam, L. J., Gee, D. G., Goff, B., Flannery, J., Telzer, E., Humphreys, K. L., et al. (2016). Stimulus-elicited connectivity influences resting-state connectivity years later in human development: a prospective study. J. Neurosci. 36, 4771-4784. doi: 10.1523/JNEUROSCI.0598-16.2016

Goldin, P. R., McRae, K., Ramel, W., and Gross, J. J. (2008). The neural bases of emotion regulation: reappraisal and suppression of negative emotion. Biol. Psychiatry 63, 577-586. doi: 10.1016/j.biopsych.2007. 05.031

Grecucci, A., Giorgetta, C., Bonini, N., and Sanfey, A. G. (2013). Reappraising social emotions: the role of inferior frontal gyrus, temporo-parietal junction and insula in interpersonal emotion regulation. Front. Hum. Neurosci. 7:523. doi: 10.3389/fnhum.2013.00523

Gross, J. J., and John, O. P. (2003). Individual differences in two emotion regulation processes: implications for affect, relationships, and well-being. J. Pers. Soc. Psychol. 85, 348-362. doi: 10.1037/0022-3514.85.2.348

Guerra-Carrillo, B., MacKey, A. P., and Bunge, S. A. (2014). Resting-state fMRI: a window into human brain plasticity. Neuroscientist 20, 522-533. doi: 10.1177/ 1073858414524442

Hartwigsen, G., Neef, N. E., Camilleri, J. A., Margulies, D. S., and Eickhoff, S. B. (2019). Functional segregation of the right inferior frontal gyrus: evidence from coactivation-based parcellation. Cereb. Cortex 29, 1532-1546. doi: 10.1093/ cercor/bhy049

Hu, T., Zhang, D., Wang, J., Mistry, R., Ran, G., and Wang, X. (2014). Relation between emotion regulation and mental health: a meta-analysis review. Psychol. Rep. 114, 341-362. doi: 10.2466/03.20.PR0.114k22w4

Hutchinson, J. B., Uncapher, M. R., Weiner, K. S., Bressler, D. W., Silver, M. A., Preston, A. R., et al. (2014). Functional heterogeneity in posterior parietal cortex across attention and episodic memory retrieval. Cereb. Cortex 24, 49-66. doi: $10.1093 /$ cercor/bhs 278

Kalisch, R. (2009). The functional neuroanatomy of reappraisal: time matters. Neurosci. Biobehav. Rev. 33, 1215-1226. doi: 10.1016/j.neubiorev.2009.06.003

Kanske, P., Heissler, J., Schönfelder, S., Bongers, A., and Wessa, M. (2011). How to regulate emotion? Neural networks for reappraisal and distraction. Cereb. Cortex 21, 1379-1388. doi: 10.1093/cercor/bhq216

Kohn, N., Eickhoff, S. B., Scheller, M., Laird, A. R., Fox, P. T., and Habel, U. (2014). Neural network of cognitive emotion regulation - an ALE meta-analysis 
and MACM analysis. Neuroimage 87, 345-355. doi: 10.1016/j.neuroimage.2013. 11.001

Kragel, P. A., Kano, M., Van Oudenhove, L., Ly, H. G., Dupont, P., Rubio, A., et al. (2018). Generalizable representations of pain, cognitive control, and negative emotion in medial frontal cortex. Nat. Neurosci. 21, 283-289. doi: 10.1038/ s41593-017-0051-7

Lane, R. D., Weihs, K. L., Herring, A., Hishaw, A., and Smith, R. (2015). Affective agnosia: expansion of the alexithymia construct and a new opportunity to integrate and extend Freud's legacy. Neurosci. Biobehav. Rev. 55, 594-611. doi: 10.1016/j.neubiorev.2015.06.007

Luks, T. L., Simpson, G. V., Dale, C. L., and Hough, M. G. (2007). Preparatory allocation of attention and adjustments in conflict processing. Neuroimage 35, 949-958. doi: 10.1016/j.neuroimage.2006.11.041

Lv, H., Wang, Z., Tong, E., Williams, L. M., Zaharchuk, G., Zeineh, M., et al. (2018). Resting-state functional MRI: everything that nonexperts have always wanted to know. Am. J. Neuroradiol. 39, 1390-1399. doi: 10.3174/ajnr.A5527

Mackey, A. P., Singley, A. T. M., and Bunge, S. A. (2013). Intensive reasoning training alters patterns of brain connectivity at rest. J. Neurosci. 33, 4796-4803. doi: 10.1523/JNEUROSCI.4141-12.2013

McRae, K., Reiman, E. M., Fort, C. L., Chen, K., and Lane, R. D. (2008). Association between trait emotional awareness and dorsal anterior cingulate activity during emotion is arousal-dependent. Neuroimage 41, 648-655. doi: 10.1016/j.neuroimage.2008.02.030

Messina, I., Bianco, S., Sambin, M., and Viviani, R. (2015). Executive and semantic processes in reappraisal of negative stimuli: insights from a meta-analysis of neuroimaging studies. Front. Psychol. 6:956. doi: 10.3389/fpsyg.2015.00956

Meyer, T., Smeets, T., Giesbrecht, T., and Merckelbach, H. (2012). The efficiency of reappraisal and expressive suppression in regulating everyday affective experiences. Psychiatry Res. 200, 964-969. doi: 10.1016/j.psychres.2012.05.034

Morawetz, C., Bode, S., Baudewig, J., and Heekeren, H. R. (2017a). Effective amygdala-prefrontal connectivity predicts individual differences in successful emotion regulation. Soc. Cogn. Affect. Neurosci. 12, 569-585. doi: 10.1093/scan/ nsw169

Morawetz, C., Bode, S., Baudewig, J., Kirilina, E., and Heekeren, H. R. (2016). Changes in effective connectivity between dorsal and ventral prefrontal regions moderate emotion regulation. Cereb. Cortex 26, 1923-1937. doi: 10.1093/ cercor/bhv005

Morawetz, C., Bode, S., Derntl, B., and Heekeren, H. R. (2017b). The effect of strategies, goals and stimulus material on the neural mechanisms of emotion regulation: a meta-analysis of fMRI studies. Neurosci. Biobehav. Rev. 72, 111128. doi: 10.1016/j.neubiorev.2016.11.014

Motes, M. A., Yezhuvath, U. S., Aslan, S., Spence, J. S., Rypma, B., and Chapman, S. B. (2018). Higher-order cognitive training effects on processing speed-related neural activity: a randomized trial. Neurobiol. Aging 62, 72-81. doi: 10.1016/j. neurobiolaging.2017.10.003

Neubauer, A. C., and Fink, A. (2009). Intelligence and neural efficiency: measures of brain activation versus measures of functional connectivity in the brain. Intelligence 37, 223-229. doi: 10.1016/j.intell.2008.10.008

Neumann, R., and Lozo, L. (2012). Priming the activation of fear and disgust: evidence for semantic processing. Emotion 12, 223-228. doi: 10.1037/a0026500

Ochsner, K. N., Silvers, J. A., and Buhle, J. T. (2012). Functional imaging studies of emotion regulation: a synthetic review and evolving model of the cognitive control of emotion. Ann. N. Y. Acad. Sci. 1251, E1-E24. doi: 10.1111/j.17496632.2012.06751.x

Olsson, A., and Ochsner, K. N. (2008). The role of social cognition in emotion. Trends Cogn. Sci. 12, 65-71. doi: 10.1016/j.tics.2007.11.010

Phillips, M. L., Ladouceur, C. D., and Drevets, W. C. (2008). A neural model of voluntary and automatic emotion regulation: implications for understanding the pathophysiology and neurodevelopment of bipolar disorder. Mol. Psychiatry 13, 833-857. doi: 10.1038/mp.2008.65

Picó-Pérez, M., Alonso, P., Contreras-Rodríguez, O., Martínez-Zalacaín, I., López-Solà, C., Jiménez-Murcia, S., et al. (2018). Dispositional use of emotion regulation strategies and resting-state cortico-limbic functional connectivity. Brain Imaging Behav. 12, 1022-1031. doi: 10.1007/s11682-0179762-3

Power, J. D., Barnes, K. A., Snyder, A. Z., Schlaggar, B. L., and Petersen, S. E. (2012). Spurious but systematic correlations in functional connectivity MRI networks arise from subject motion. Neuroimage 59, 2142-2154. doi: 10.1016/j. neuroimage.2011.10.018

Salo, E., Salmela, V., Salmi, J., Numminen, J., and Alho, K. (2017). Brain activity associated with selective attention, divided attention and distraction. Brain Res. 1664, 25-36. doi: 10.1016/j.brainres.2017.03.021

Sang, N., Zhang, L., Hao, L., Wang, Y., Wang, X., Zhang, F., et al. (2017). Human sensory cortex structure and top-down controlling brain network determine individual differences in perceptual alternations. Neurosci. Lett. 636, 113-119. doi: 10.1016/j.neulet.2016.10.048

Schulz, K. P., Clerkin, S. M., Halperin, J. M., Newcorn, J. H., Tang, C. Y., and Fan, J. (2009). Dissociable neural effects of stimulus valence and preceding context during the inhibition of responses to emotional faces. Hum. Brain Mapp. 30, 2821-2833. doi: 10.1002/hbm.20706

Smith, S. M., Fox, P. T., Miller, K. L., Glahn, D. C., Fox, P. M., Mackay, C. E., et al. (2009). Correspondence of the brain's functional architecture during activation and rest. Proc. Natl. Acad. Sci. U.S.A. 106, 13040-13045. doi: 10.1073/pnas. 0905267106

Uchida, M., Biederman, J., Gabrieli, J. D. E., Micco, J., De Los Angeles, C., Brown, A., et al. (2014). Emotion regulation ability varies in relation to intrinsic functional brain architecture. Soc. Cogn. Affect. Neurosci. 10, 1738-1748. doi: $10.1093 /$ scan/nsv059

van Dijk, K. R. A., Sabuncu, M. R., and Buckner, R. L. (2012). The influence of head motion on intrinsic functional connectivity MRI. Neuroimage 59, 431-438. doi: 10.1016/j.neuroimage.2011.07.044

Vartanian, O., Jobidon, M. E., Bouak, F., Nakashima, A., Smith, I., Lam, Q., et al. (2013). Working memory training is associated with lower prefrontal cortex activation in a divergent thinking task. Neuroscience 236, 186-194. doi: 10.1016/ j.neuroscience.2012.12.060

Wager, T. D., Davidson, M. L., Hughes, B. L., Lindquist, M. A., and Ochsner, K. N. (2008). Prefrontal-subcortical pathways mediating successful emotion regulation. Neuron 59, 1037-1050. doi: 10.1016/j.neuron.2008.09.006

Wang, M., Yu, B., Luo, C., Fogelson, N., Zhang, J., Jin, Z., et al. (2020). Evaluating the causal contribution of fronto-parietal cortices to the control of the bottomup and top-down visual attention using fMRI-guided TMS. Cortex 126, $200-$ 212. doi: 10.1016/j.cortex.2020.01.005

Webb, T. L., Miles, E., and Sheeran, P. (2012). Dealing with feeling: a meta-analysis of the effectiveness of strategies derived from the process model of emotion regulation. Psychol. Bull. 138, 775-808. doi: 10.1037/a0027600

Xie, K., Jin, Z., Ni, X., Zhang, J., and Li, L. (2020). Distinct neural substrates underlying target facilitation and distractor suppression: a combined voxel-based morphometry and resting-state functional connectivity study. Neuroimage 221:117149. doi: 10.1016/j.neuroimage.2020.117149

Yan, C. G., Yang, Z., Colcombe, S. J., Zuo, X. N., and Milham, M. P. (2017). Concordance among indices of intrinsic brain function: insights from interindividual variation and temporal dynamics. Sci. Bull. 62, 1572-1584. doi: 10. 1016/j.scib.2017.09.015

Zaehringer, J., Jennen-Steinmetz, C., Schmahl, C., Ende, G., and Paret, C. (2020). Psychophysiological effects of downregulating negative emotions: insights from a meta-analysis of healthy adults. Front. Psychol. 11:470. doi: 10.3389/fpsyg. 2020.00470

Zhang, Q., Wang, H., Luo, C., Zhang, J., Jin, Z., and Li, L. (2019). The neural basis of semantic cognition in Mandarin Chinese: a combined fMRI and TMS study. Hum. Brain Mapp. 40, 5412-5423. doi: 10.1002/hbm.24781

Zuo, X. N., Ehmke, R., Mennes, M., Imperati, D., Castellanos, F. X., Sporns, O., et al. (2012). Network centrality in the human functional connectome. Cereb. Cortex 22, 1862-1875. doi: 10.1093/cercor/bhr269

Conflict of Interest: The authors declare that the research was conducted in the absence of any commercial or financial relationships that could be construed as a potential conflict of interest.

Copyright (c) 2021 Li, Xie, Ngetich, Zhang, Jin and Li. This is an open-access article distributed under the terms of the Creative Commons Attribution License (CC BY). The use, distribution or reproduction in other forums is permitted, provided the original author(s) and the copyright owner(s) are credited and that the original publication in this journal is cited, in accordance with accepted academic practice. No use, distribution or reproduction is permitted which does not comply with these terms. 